\title{
Compensation defects in annealed undoped liquid encapsulated Czochralski InP
}

\author{
S. Fung, ${ }^{\text {a) }}$ Y. W. Zhao, X. L. Xu, and X. D. Chen \\ Department of Physics, The University of Hong Kong, Hong Kong People's Republic of China \\ N. F. Sun, T. N. Sun, R. G. Zhang, S. L. Liu, and G. Y. Yang \\ Hebei Semiconductor Research Institute, P.O. Box 179, Shijiazhuang, Hebei, People's Republic of China \\ X. B. Guo, Y. Z. Sun, R. Y. Yan, and Q. H. Hua \\ Tianjin Semiconductor Institute, P.O. Box 55, Tianjin, People's Republic of China
}

(Received 4 June 1998; accepted for publication 9 April 1999)

\begin{abstract}
As-grown undoped $n$-type semiconducting and annealed undoped semi-insulating (SI) liquid encapsulated Czochralski (LEC) InP has been studied by temperature dependent Hall measurement, photoluminescence spectroscopy, infrared absorption, and photocurrent spectroscopy. $P$-type conduction SI InP can frequently be obtained by annealing undoped LEC InP. This is caused by a high concentration of thermally induced native acceptor defects. In some cases, it can be shown that the thermally induced $n$-type SI property of undoped LEC InP is caused by a midgap donor compensating for the net shallow acceptors. The midgap donor is proposed to be a phosphorus antisite related defect. Traps in annealed SI InP have been detected by photocurrent spectroscopy and have been compared with reported results. The mechanisms of defect formation are discussed.
\end{abstract}

(C) 1999 American Institute of Physics. [S0021-8979(99)03014-5]

\section{INTRODUCTION}

Semi-insulating (SI) liquid encapsulated Czochralski (LEC) InP is usually obtained by doping with a high concentration $\left(\geqslant 10^{16} \mathrm{~cm}^{-3}\right)$ of $\mathrm{Fe}^{1,2}$ The outdiffusion of $\mathrm{Fe}$ from the substrate to the epitaxy layer is detrimental to the quality of InP based devices. ${ }^{3}$ Thus, an iron-free undoped SI InP substrate is preferred. Unfortunately, undoped or even low Fe concentration $\left(\leqslant 10^{15} \mathrm{~cm}^{-3}\right)$ doped as-grown LEC SI InP has rarely been reported although high purity polycrystalline InP was used.

In 1986, undoped LEC InP was found to change into high resistivity materials $\left(3.6 \times 10^{5} \Omega \mathrm{cm}\right)$ by high temperature phosphorus ambient annealing for three weeks. ${ }^{4}$ SI (with resistivity of $\sim 10^{6}-10^{7} \Omega \mathrm{cm}$ ) undoped InP was obtained by annealing high purity undoped LEC InP. ${ }^{5-9}$ The compensation mechanism in annealed SI InP has also been studied extensively. ${ }^{8-13}$ The main question here is whether this annealed SI property is caused by thermally induced native defects or by inadvertent and residual deep level metal contamination. Fe contamination or activation in a low concentration of $10^{15} \mathrm{~cm}^{-3}$ has been observed in annealed undoped SI InP sample. ${ }^{6,10}$ Lightly Fe-doped $n$-type semiconducting LEC InP was also found to be annealed into SI material. ${ }^{14,15}$ All these phenomena indicated that residual Fe in the material was probably responsible for the thermally induced SI property.

With electron spin resonance and calorimetric absorption spectroscopy measurements, ${ }^{10}$ no $\mathrm{Fe}^{3+}$ was detected in annealed undoped SI InP, indicating that all residual Fe in this material was in the $\mathrm{Fe}^{2+}$ state, as distinct from Fe-doped SI

${ }^{a)}$ Electronic mail: sfung@ @kucc.hku.hk
InP in which both $\mathrm{Fe}^{2+}$ and $\mathrm{Fe}^{3+}$ can be detected. ${ }^{16,17} \mathrm{~A}$ few defects with energy levels in the range of $0.2-0.6 \mathrm{eV}$ have also been found in annealed SI InP which is either undoped or lightly Fe doped. ${ }^{14,15}$ These results indicate that some native defects, which may also be involved in the compensation mechanism, have formed after the annealing process.

In this article, annealed undoped LEC InP is characterized by room temperature Hall, temperature dependent Hall (TDH) measurements, photoluminescence (PL) spectroscopy, and infrared absorption. Defects in undoped SI InP are detected by room temperature photocurrent spectroscopy and compared with the results of other deep level spectroscopy measurements. Compensation in annealed undoped SI InP will be discussed.

\section{EXPERIMENT}

All samples are undoped InP wafers sliced from ingots which are grown, by an in situ injection synthesis LEC method in one of our laboratories. ${ }^{18}$ Annealing of LEC undoped $n$-type InP wafers is carried out in a sealed quartz tube with about 60 mbar phosphorus ambient. The annealing temperature is usually maintained at $900{ }^{\circ} \mathrm{C}$ and the sample is cooled slowly to room temperature after annealing. Room temperature Hall measurements and TDH measurements are carried out on samples of van der Pauw configuration using a magnetic field of $5 \mathrm{kG}$. After annealing, a layer of at least 80 $\mu \mathrm{m}$ on both sides of the wafer is removed to ensure proper bulk property measurements. Single-side-polished samples are used for the PL measurement while double-side-polished wafers are used for infrared absorption experiments. The PL system consists of a $480 \mathrm{~nm}$ argon laser excitation source, a Spex $1 \mathrm{~m}$ grating spectrometer, and a photomultiplier Infra- 
TABLE I. Electrical parameters of undoped LEC InP after annealing at $900{ }^{\circ} \mathrm{C}$ for $90 \mathrm{~h}$.

\begin{tabular}{cccccccc}
\hline \hline & \multicolumn{3}{c}{ Before annealing } & & \multicolumn{3}{c}{ After annealing } \\
\cline { 2 - 4 } Sample No. & $n\left(\mathrm{~cm}^{-3}\right)$ & $\mu\left(\mathrm{cm}^{2} / \mathrm{V} \mathrm{s}\right)$ & $\rho(\Omega \mathrm{cm})$ & & $n\left(\mathrm{~cm}^{-3}\right)$ & $\mu\left(\mathrm{cm}^{2} / \mathrm{V} \mathrm{s}\right)$ & $\rho(\Omega \mathrm{cm})$ \\
\hline A-N-01 & $3.42 \times 10^{15}$ & 3740 & 0.49 & & $8.37 \times 10^{7}$ & 2710 & $2.76 \times 10^{7}$ \\
& & & & & $2.34 \times 10^{11}$ & 16 & $1.24 \times 10^{7}$ \\
A-N-02 & $4.29 \times 10^{15}$ & 4090 & & & $2.11 \times 10^{11}$ & 28 & $1.05 \times 10^{6}$ \\
A-N-03 & $5.33 \times 10^{15}$ & 4320 & & & $2.45 \times 10^{10}$ & 40 & $6.36 \times 10^{6}$ \\
A-N-04 & $4.13 \times 10^{15}$ & 4620 & & & $2.07 \times 10^{11}$ & 969 & $3.12 \times 10^{4}$ \\
A-N-05 & $1.07 \times 10^{16}$ & 4090 & & & $7.43 \times 10^{15}$ & 3420 & 0.24 \\
A-N-06 & $1.31 \times 10^{16}$ & 3790 & 0.13 & & $8.43 \times 10^{15}$ & 3330 & 0.22 \\
& & & & & $4.05 \times 10^{14}$ & 4550 & 3.39 \\
B-N-01 & $4.88 \times 10^{15}$ & 3790 & 0.34 & & $6.86 \times 10^{15}$ & 1340 & 0.68 \\
B-N-02 & $2.40 \times 10^{15}$ & 3280 & 0.18 & & $4.14 \times 10^{15}$ & 1830 & 8.26 \\
\hline \hline
\end{tabular}

red absorption measurements are carried out on a Brucker ISF 120 Fourier transform infrared absorption spectrometer. Room temperature photocurrent spectroscopy measurements are carried out on single-side-polished SI InP samples in the photon energy range of $0.3-1.6 \mathrm{eV}$. The system used for the photocurrent spectroscopy measurement is described elsewhere. ${ }^{19}$ The sample size is $5 \times 10 \mathrm{~mm}$ and the applied bias is $100 \mathrm{~V}$ for these measurements. AuGe alloy is used for making the necessary ohmic contacts.

\section{RESULTS}

\section{A. Electrical measurements}

All annealing results of undoped LEC InP are summarized in Table I. The electrical parameters of annealed InP change in two different ways. Undoped InP with a low carrier concentration becomes SI, whereas high carrier concentration materials cannot be made SI by annealing; only a reduction in the net electron concentration upon annealing can be achieved. As shown in Table I, SI InP material with very low mobility is obtained frequently. This low mobility is an indication of mixed conduction in the material. For mixed conduction material, the Hall coefficient and mobility can be expressed as follows: ${ }^{20}$

$$
\begin{aligned}
& R_{H}=\frac{r_{H}}{q} \frac{\left[n-p\left(\frac{\mu_{n}}{\mu_{p}}\right)^{2}\right]}{\left[p+n\left(\frac{\mu_{n}}{\mu_{p}}\right)^{2}\right]}, \\
& \mu_{H}=r_{H} \mu_{n} \frac{\left[n-p\left(\frac{\mu_{p}}{\mu_{n}}\right)^{2}\right]}{\left[n+p\left(\frac{\mu_{p}}{\mu_{n}}\right)^{2}\right]},
\end{aligned}
$$

where $n$ is the electron concentration and $p$ is the hole concentration. $\mu_{n}$ and $\mu_{p}$ are the mobilities of the electrons and holes, respectively. Since the ratio of $\mu_{n}$ to $\mu_{p}$ of InP is about 30, from Eqs. (1) and (2) the electron concentration is about $(7-36) \times 10^{9} \mathrm{~cm}^{-3}$, and the hole concentration is about $(5.6-28) \times 10^{12} \mathrm{~cm}^{-3}$, implying that mixed conduction materials are $p$ type semi-insulating.

The TDH measurement result of sample A-N-01 is shown in Fig. 1(b). The temperature range of this measure- ment is $6-300 \mathrm{~K}$ before annealing and $300-430 \mathrm{~K}$ after annealing. From Fig. 1(b), it can be seen that the carrier concentration saturates at room temperature and impurity band conduction appears at low temperature. It is a typical TDH result of compensated $n$-type InP. ${ }^{21}$ The result also indicates that the concentration of deep levels is low and that the compensation mainly comes from shallow levels in the material. From the carrier concentration change in the temperature range of $70-100 \mathrm{~K}$, the ionization energy of the donors in this sample can be deduced by fitting the following well known equation:

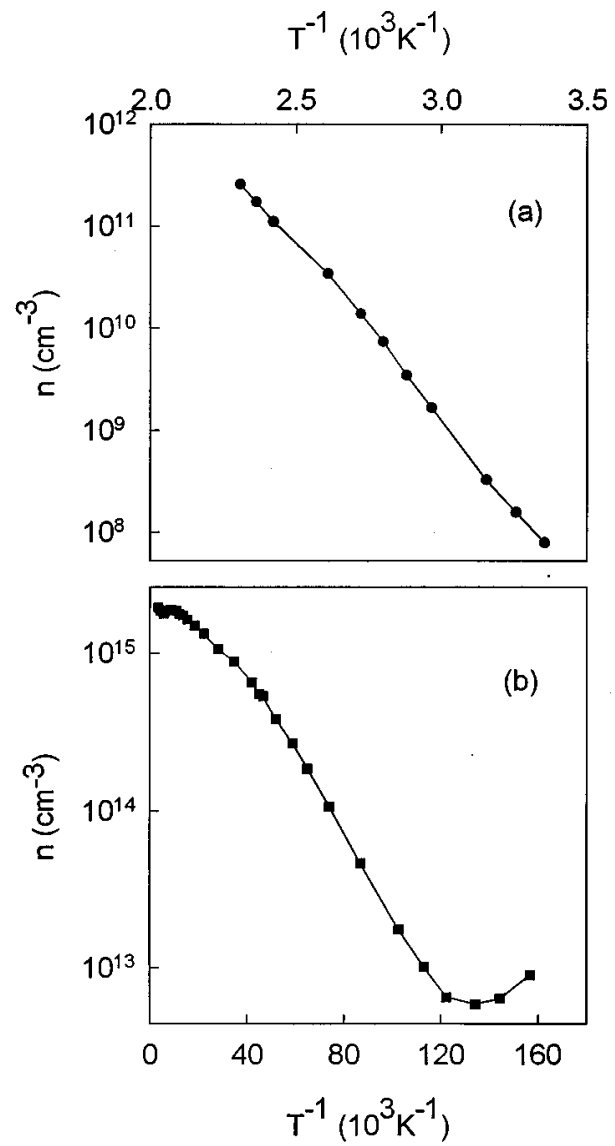

FIG. 1. Temperature dependent Hall measurements of sample A-N-01. (a) After annealing; (b) before annealing. 


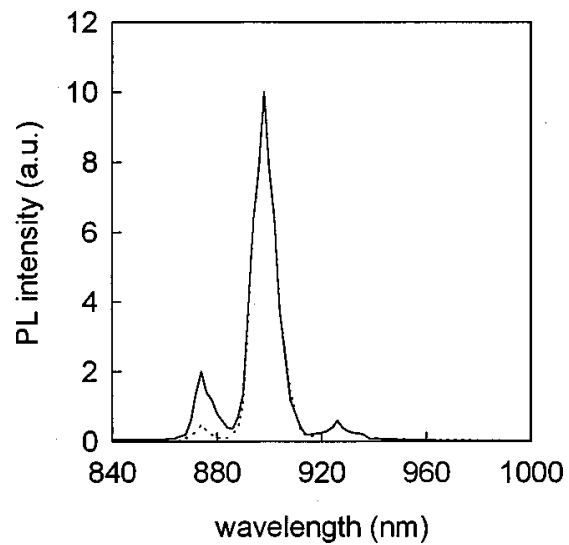

FIG. 2. PL spectra taken at $19 \mathrm{~K}$ for sample A-N-01. The solid line is before annealing and the dashed line is after annealing.

$$
\frac{n\left(n+N_{A}\right)}{N_{D}-N_{A}-n}=\frac{1}{g} N_{c} e^{-E_{D} / k T},
$$

where the symbols have their usual meanings. The result is $N_{D}=1.2 \times 10^{16} \mathrm{~cm}^{-3}, \quad N_{A}=8.5 \times 10^{15} \mathrm{~cm}^{-3}, \quad$ and $E_{D}$ $=7 \mathrm{meV}$. Thus, the dominant donor level in the material is shallow $(=7 \mathrm{meV})$.

After annealing, the sample becomes SI and the TDH measurement is carried out in the temperature range of 300$430 \mathrm{~K}$, as shown in Fig. 1(a). The carrier activation energy is obtained from the slope of $n T^{-1.5}$ vs $1 / k T$ and is found to be $0.66 \mathrm{eV}$. Thus, a high concentration of midgap levels must exist and compensate for shallow levels in the annealed sample and give rise to the semi-insulating property.

\section{B. Optical measurements}

Sample A-N-01 has also been studied by photoluminescence spectroscopy and infrared absorption spectroscopy at around $20 \mathrm{~K}$. The spectra are shown in Figs. 2 and 3, respec-

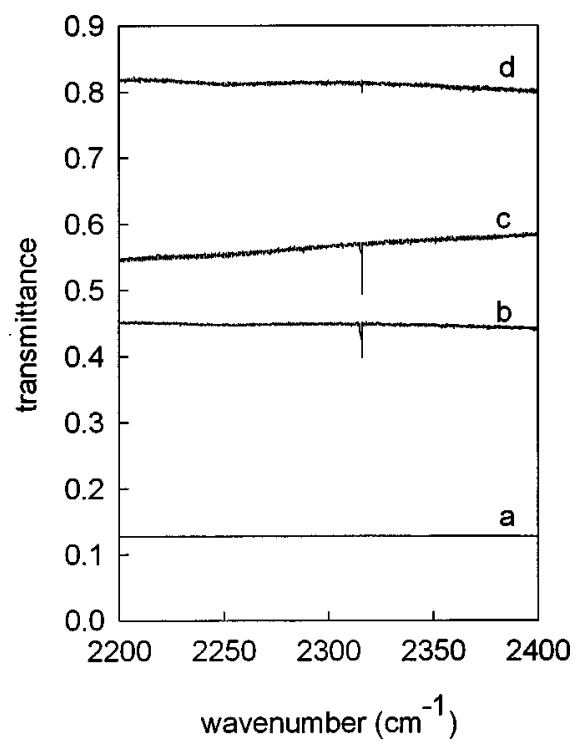

FIG. 3. Low temperature infrared transmission spectra of LEC InP after annealing (a) A-N-01, $17 \mathrm{~K}$; and before annealing (b) A-N-01, $33 \mathrm{~K}$; (c) Fe-doped SI InP, 32 K; (d) B-N-01, 22 K. tively. As shown in Fig. 2, there is a strong peak at a photon energy of about $1.38 \mathrm{eV}$. This structure has been shown to be due to the transition from the conduction band to a shallow acceptor level in InP. ${ }^{22}$ This is confirmation of a high concentration of shallow acceptor impurities in the material. After annealing, the PL spectrum is nearly unchanged, indicating a negligible influence of the heat treatment process on the acceptor impurity concentration.

Figure 3 gives the spectra of infrared absorption in the range of $2200-2400 \mathrm{~cm}^{-1}$, indicating the presence of a hydrogen indium vacancy complex $V_{\mathrm{In}} \mathrm{H}_{4}$ whose local vibration frequency is around $2316 \mathrm{~cm}^{-1}$ at low temperature. ${ }^{23}$ It is seen in Fig. 3 that $V_{\text {In }} \mathrm{H}_{4}$ can be detected by infrared absorption in both undoped $n$ type and Fe-doped SI LEC InP. The weak absorption peak of sample B-N-01 indicates that the concentration of this defect in this particular sample is low. The concentration of $V_{\mathrm{In}} \mathrm{H}_{4}$, estimated by the calibration data of the $\mathrm{Zn}-\mathrm{H}$ complex in $\mathrm{InP},{ }^{24,25}$ is in the range of $10^{15}-10^{16} \mathrm{~cm}^{-3}$. In sample B-N-01 it is at least five times smaller. The disappearance of the infrared absorption peak of $V_{\text {In }} \mathrm{H}_{4}$ after annealing [trace (a)] is evidence that it is annihilated in the annealing process.

\section{Transient photocurrent measurements}

Photocurrent spectroscopy measurements at room temperature were done for annealed undoped $n$ type and mixed conduction SI InP samples. For comparison, photocurrent spectra are also given for a Fe-doped SI InP sample annealed at different temperatures. The defect levels can be identified from the transient current peaks produced by the optical excitation process. The spectra shown in Fig. 4 indicate several trap levels exist in the band gap. These are at $0.43-0.77 \mathrm{eV}$ from the respective band edges. Note that this technique cannot determine which band is involved in the transition. In mixed conduction SI InP, similar trap levels are detected, but their concentrations are different.

Two transient current peaks at 0.65 and $0.66 \mathrm{eV}$ can be seen in the spectra of annealed undoped and Fe-doped SI InP. In contrast to that, in all our as-grown (nonannealed) Fe-doped SI InP samples, the only Fe acceptor level detected is at $0.66 \mathrm{eV}$. From the spectra of Fe-doped InP annealed at 600 and $700{ }^{\circ} \mathrm{C}(30 \mathrm{~min}$ and $12 \mathrm{~h}$ ), it can be seen that the intensity of the $0.65 \mathrm{eV}$ peak increases with the annealing time. Moreover, along with the increasing intensity of the $0.65 \mathrm{eV}$ a peak at $1.29 \mathrm{eV}$ appears in the spectra of annealed Fe-doped InP and undoped $n$-type SI InP. Thus the $1.29 \mathrm{eV}$ peak seems to correlate with the $0.65 \mathrm{eV}$ peak in the annealed SI InP. We assign this feature to the electron transition from the $0.65 \mathrm{eV}$ level to one of the higher conduction band minima, say, the $L$ point. This assignment implies that both transitions involve the same original ground state. We note that the $1.29 \mathrm{eV}$ peak is not observed in as-grown (nonannealed) Fe-doped SI InP material. Based on these observations, it can be concluded that the $0.65 \mathrm{eV}$ midgap level is most probably a thermally induced defect in the annealed SI InP material. 


\section{$E(e V)$}
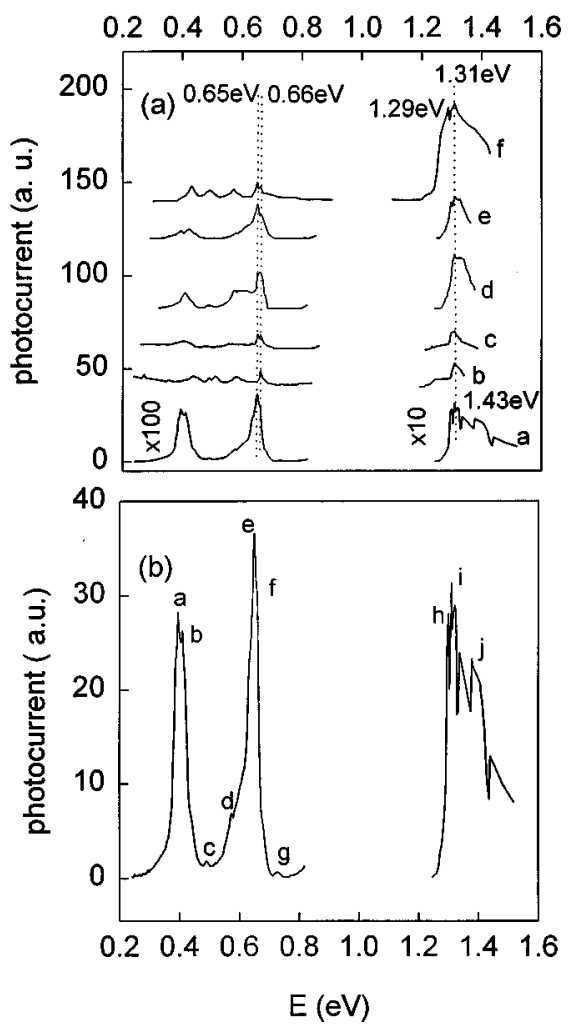

FIG. 4. Room temperature photocurrent spectra of annealed SI InP. (a) Undoped, Fe-doped and mixed conduction semi-insulating InP; (b) annealed undoped semi-insulating and InP. In (a), the semi-insulating samples were subjected to different annealing conditions: a) undoped $n$ type SI; b) asgrown $\mathrm{Fe}$ doped; c) $\mathrm{Fe}$ doped annealed at $600^{\circ} \mathrm{C}, 30 \mathrm{~min}$; c) $\mathrm{Fe}$ doped annealed at $700{ }^{\circ} \mathrm{C}, 30 \mathrm{~min}$; d) $\mathrm{Fe}$ doped annealed at $700{ }^{\circ} \mathrm{C}, 12 \mathrm{~h}$; e) annealed undoped mixed conduction SI. The energy positions of peaks $\mathrm{a}-\mathrm{j}$ in (b) are $0.43,0.44,0.49,0.57,0.65,0.66,0.77,1.30,1.31$, and $1.43 \mathrm{eV}$, respectively.

\section{DISCUSSION}

Both electrical and photocurrent measurements of annealed undoped SI InP have shown that the electrical changes are closely related to the formation of thermally produced defects in the material. So far, knowledge about the formation process of defects in annealed InP is incomplete. A previously proposed model ${ }^{26}$ may be helpful for interpretation of our results. This model is as follows:

$$
\begin{aligned}
& 4 V_{\mathrm{In}} \mathrm{H}_{4}+6 e \rightarrow V_{\mathrm{In}} \mathrm{H}_{3}+V_{\mathrm{In}} \mathrm{H}_{2}^{2-}+V_{\mathrm{In}} \mathrm{H}^{1-}+V_{\mathrm{In}}^{3-}+10 \mathrm{H}^{0} \uparrow \\
& \mathrm{P}+V_{\mathrm{In}}^{3-} \rightarrow P_{\mathrm{In}}^{2+}+V_{\mathrm{P}}^{+}+6 e \\
& \mathrm{In}+V_{\mathrm{P}}^{+}+6 e \rightarrow \mathrm{In}_{\mathrm{P}}^{2-}+V_{\mathrm{In}}^{3-}
\end{aligned}
$$

$V_{\text {In }} \mathrm{H}_{4}$ is a common defect in as-grown LEC InP as evidenced by the infrared absorption spectra shown in Fig. 3. The concentration has been found to depend on the compensation ratio. $^{27}$ The disappearance of the absorption line in the spectrum of annealed InP [Fig. 3, trace (a)] indicates that the defect is annihilated in the annealing process. According to Eq. (4), after the annihilation, indium vacancy related defects are left behind acting as precursors for the formation of other defects [shown in Eqs. (5) and (6)]. According to Table I, $B$ type samples have a much lower $V_{\text {In }} \mathrm{H}_{4}$ concentration than $A$ type samples. After annealing, the change in electrical parameters for the $A$ type samples is relatively large, indicating the important role of this complex in the compensation process.

Based on these defect evolution equations, a tentative assignment of those defects detected by photocurrent spectroscopy can be given. The $0.43 \mathrm{eV}$ peak in Fig. 4 is a common defect produced by high temperature annealing of LEC InP. A similar level has been measured in annealed undoped SI InP by photoinduced current transient spectroscopy $(\mathrm{PICTS})^{28}$ and in annealed low resistivity InP by deep level transient spectroscopy (DLTS). ${ }^{13}$ This level is usually considered to be a phosphorus vacancy related defect and related to phosphorus deficiency. Close to this peak, a peak at 0.44 $\mathrm{eV}$ possibly results from the excitation of a residual deep level impurity such as $\mathrm{Cr}^{29}$ The 0.49 and $0.57 \mathrm{eV}$ levels are close to the PICTS levels labeled $A_{4}$ and $A_{5}$ by Marrakchi et $a{ }^{28}{ }^{28}$ These two levels were considered to be related to phosphorus overpressure, thus they are possibly related to an indium vacancy. A level similar to the $0.77 \mathrm{eV}$ level has been found in annealed $n$-type and $p$-type LEC InP by a photocapacitance (PHCAP) measurement by Nishizawa et $a l$. and is assigned to the excitation of holes from a defect level to the valence band, found by the PHCAP result. ${ }^{30,31}$ There is a possibility that the 0.77 and $0.57 \mathrm{eV}$ peaks are excitation of holes and electrons from one defect level to the valence band and the conduction band, respectively.

With regard to the $0.65 \mathrm{eV}$ midgap defect level, its assignment to a phosphorus antisite related defect is consistent with a calculation showing that this defect should indeed be located at the middle of the gap. ${ }^{32}$ Experimental studies have also found a midgap defect level related to a phosphorus antisite in InP. ${ }^{33}$ Recently a midgap level at $E_{c}-0.64 \mathrm{eV}$ was also found in annealed $n$-type LEC InP by PHCAP. ${ }^{30}$ Its concentration can be suppressed by increasing the amphoteric impurity concentration, indicating that it is a native defect. High concentrations of phosphorus antisite defects were detected by optically detected magnetic resonance only in annealed undoped high resistivity LEC InP. ${ }^{34}$

There seems to be a relationship between the thermally induced 0.43 and $0.65 \mathrm{eV}$ levels, since both appear in one annealed Fe-doped sample and neither can be seen in other samples. These observations support the suggestion that they are due to thermally induced defects rather than to inadvertent metal contamination. The presence of a high concentration of shallow acceptors in the material is qualitatively confirmed by the PL measurement and this is in agreement with a previous mass spectrometer analysis. ${ }^{25}$ The assignment of the $0.66 \mathrm{eV}$ photocurrent peak to a Fe related acceptor level is consistent with the observation that it is very weak in annealed $n$-type SI InP which is known to possess a very low Fe concentration. We conclude that in annealed undoped SI InP the compensation center at the $0.65 \mathrm{eV}$ level is a native defect rather than a Fe acceptor level.

\section{CONCLUSION}

The electrical properties of undoped LEC InP can be changed significantly by annealing at high temperature. $N$ 
type and mixed conduction (with $p$ type) semi-insulating undoped InP can be produced upon annealing. The acceptor concentration is mainly increased by native defects. The higher compensation level causes a high purity material to change from $n$ type to $p$ type. A midgap defect forms and is attributed to a phosphorus antisite related defect. A defect level at $0.43 \mathrm{eV}$ is generated simultaneously.

\section{ACKNOWLEDGMENT}

One of the authors (S.F.) wishes to acknowledge valuable financial support from the Hong Kong University CRCG and the Hong Kong RGC research grants.

${ }^{1}$ D. Rumsby, R. M. Ware, and M. Whittaker, Semi-insulating III-V Materials, Nottingham, edited by G. J. R. Rees (Shiva, Nantwich, 1980), p. 59.

${ }^{2}$ K. Kainosho, H. Shimakura, T. Kanazawa, T. Inoue, and O. Oda, in Proceedings of the 16th International Symposium on GaAs and Related Compounds, Karuizawa, edited by T. Ikoma and H. Watanabe (Institute of Physics, New York, 1990), p. 25.

${ }^{3}$ D. E. Holmes, R. G. Wilson, and P. W. Yu, J. Appl. Phys. 52, 3396 (1981).

${ }^{4}$ P. B. Klein, R. L. Henry, T. A. Kennedy, and N. D. Wilsey, Mater. Sci. Forum 10-12, 1259 (1986).

${ }^{5}$ D. Hofmann, G. Müller, and N. Streckfuss, Appl. Phys. A: Solids Surf. 48, 315 (1989).

${ }^{6}$ G. Hirt, D. Hofmann, F. Mosel, N. Schafer, and G. Müller, J. Electron. Mater. 20, 1065 (1991)

${ }^{7}$ K. Kainosho, H. Shimakura, H. Yamamoto, and O. Oda, Appl. Phys. Lett. 59, 932 (1991)

${ }^{8}$ R. Fornari, A. Brinciotti, E. Gombia, R. Mosca, and A. Sentiri, Mater. Sci. Eng., B 28, 95 (1994).

${ }^{9}$ A. Hruban, St. Strzelecka, W. Wegner, M. Gladysz, W. Orlowski, M. Piesa, and A. Mirowska, Proceedings of the 8th Conference on Semiinsulating III-V Materials, Warsaw, Poland 1994, edited by M. Goldlewski (World Scientific, Singapore, 1994), p. 43.

${ }^{10}$ T. Wolf, D. Bimberg, G. Hirt, D. Hofmann, and G. Müller, Proceedings of the 4th International Conference on InP and Related Materials, Newport, 1992, p. 630 (IEEE, New York, 1992).

${ }^{11}$ G. Hirt, T. Mono, and G. Müller, Mater. Sci. Eng., B 28, 101 (1994).

${ }^{12}$ G. Hirt, D. Wolf, and G. Müller, J. Appl. Phys. 74, 5583 (1993).
${ }^{13}$ P. Kipfer, J. Lindolf, D. Hofmann, and G. Müller, J. Appl. Phys. 69, 3860 (1991).

${ }^{14}$ K. Kainosho, O. Oda, G. Hirt, and G. Müller, Mater. Res. Soc. Symp. Proc. 325, 101 (1994).

${ }^{15}$ R. Fornari, A. Zappettini, E. Gombia, R. Mosca, K. Cherkaoui, and G. Marrakchi, J. Appl. Phys. 81, 7604 (1997).

${ }^{16}$ W. H. Koschel, U. Kaufmann, and S. G. Bishop, Solid State Commun. 21, 1069 (1977).

${ }^{17}$ A. Juhl, A. Hoffmann, D. Bimberg, and H. Schulz, Appl. Phys. Lett. 50, 1292 (1987)

${ }^{18} \mathrm{~S}$. Tong-nien, L. Szu-lin, and K. Shu-tseng, Proceedings of the 2nd Conference on Semi-insulating III-V Materials, Evian, France 1982, edited by S. Makram-Ebeid and B. Tuck (Shiva, Nantwich, England, 1982), p. 61.

${ }^{19} \mathrm{~K} . \mathrm{Hu}, \mathrm{X}$. Guo, Z. Zhou, M. Zhang, G. Li, and X. He, in Proceedings of the 4th International Conference on Solid-State and Integrated-Circuit Technology, edited by G. L. Baldwin, Z. Li, C. C. Tsai, and J. Zhang (The Electronica Industry, Beijing, 1995), p. 521.

${ }^{20}$ R. Zucca, J. Appl. Phys. 48, 1987 (1977).

${ }^{21}$ P. Blood and J. W. Orton, J. Phys. C 7, 803 (1974).

${ }^{22}$ E. Kubota, Y. Ohmori, and K. Sugii, J. Appl. Phys. 55, 3779 (1984).

${ }^{23}$ R. Darwich, B. Pajot, B. Rose, D. Robein, B. Theys, R. Rahbi, C. Porte, and F. Gendron, Phys. Rev. B 48, 17776 (1993).

${ }^{24}$ B. Clerjaud, D. Côte, C. Naud, M. Gauneau, and R. Chaplain, Appl. Phys. Lett. 59, 2980 (1990).

${ }^{25}$ C. P. Ewels, S. Öberg, R. Jones, B. Pajot, and P. R. Briddon, Semicond. Sci. Technol. 11, 502 (1996).

${ }^{26}$ Y. W. Zhao et al., Appl. Phys. Lett. 70, 2126 (1998).

${ }^{27}$ S. Fung et al., Appl. Phys. Lett. 73, 1275 (1998).

${ }^{28}$ G. Marrakchi, K. Cherkaoui, A. Karoui, G. Hirt, and G. Müller, J. Appl. Phys. 79, 6947 (1996).

${ }^{29}$ S. Fung and R. J. Nicholas, J. Phys. C 14, 2135 (1981).

${ }^{30}$ J. Nishizawa, Y. Oyama, K. Suto, and K. Kim, J. Appl. Phys. 80, 1488 (1996).

${ }^{31}$ J. Nishizawa, K. Kim, Y. Oyama, and K. Suto, J. Appl. Phys. 81, 3151 (1996).

${ }^{32}$ A. P. Seitsonen, R. Virkkunen, M. J. Puska, and R. M. Nieminen, Phys. Rev. B 49, 5253 (1994).

${ }^{33}$ H. P. Gislason, in Semi-insulating III-V Materials, edited by G. Grossmann and L. Ledebo (Institute of Physics, New York, 1998), p. 311.

${ }^{34}$ T. A. Kennedy, N. D. Wisey, P. B. Klein, and R. L. Henry, Mater. Sci. Forum 10-12, 271 (1986). 